\title{
Everything in excess is opposed to nature, even vitamin D: a case report
}

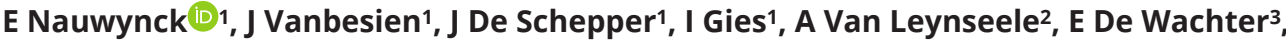 \\ B Hauser ${ }^{4}$ and W Staels ${ }^{1}$ \\ 1Division of Pediatric Endocrinology, KidZ Health Castle, UZ Brussel, Vrije Universiteit Brussel, Brussels, Belgium, \\ ${ }^{2}$ Department of Pediatrics, AZ Jan Portaels, Vilvoorde, Belgium, ${ }^{3}$ Division of Pediatric Pulmonology, and ${ }^{4}$ Division of \\ Pediatric Gastroenterology, KidZ Health Castle, UZ Brussel, Vrije Universiteit Brussel, Brussels, Belgium
}

\author{
Correspondence \\ should be addressed \\ to E Nauwynck \\ Email \\ enauwynck@gmail.com
}

\section{Summary}

Vitamin $D$ intoxication in children is rare but its incidence is increasing as vitamin $D$ is supplemented more often and in higher doses. Children with cystic fibrosis (CF) are at risk for vitamin D intoxication due to incorrect compounded preparations of liposoluble vitamins. Here, we report a severe vitamin D intoxication in a 4-year-old girl with CF, due to an error in the compounded vitamin A, D, E, and K preparation, presenting clinically with weight loss, constipation, polydipsia, polyuria, and nycturia. The administered compounded preparation contained 10000 -fold the prescribed vitamin D dose. The patient was treated with hyperhydration, loop diuretics, and bisphosphonates. Serum calcium levels normalized after 4 days but serum 25-hydroxyvitamin D levels remained elevated even up to 2 months after treatment.

\section{Learning points:}

- Vitamin D intoxication should be ruled out when patients with cystic fibrosis (CF) present with acute polyuria, constipation, and weight loss.

- Prompt treatment is necessary to avert life-threatening complications.

- Regularly measuring serum calcium and 25-hydroxyvitamin D concentrations in children with CF receiving vitamin $A, D, E$, and $K$ supplements is important during their follow-up.

\section{Background}

Although vitamin D intoxication is uncommon in children, it requires a rapid diagnosis to avoid severe acute and long-term complications of hypercalcemia. Coma, ventricular fibrillation, and acute renal insufficiency are possible life-threatening presentations of vitamin D-induced hypercalcemia (1). Nephrocalcinosis, renal lithiasis, vascular calcifications, and arterial hypertension are known late complications of severe or longstanding hypercalcemia $(2,3)$. Vitamin D intoxication should be suspected in every child receiving vitamin D supplements when complaining of vague abdominal symptoms, fatigue, and polydipsia - the classical presenting symptoms of hypercalcemia.
The most common causes of vitamin D intoxication in children are manufacturing errors, parental dosing errors, and erroneous medical prescriptions $(2,4,5,6,7)$. Children with cystic fibrosis (CF) are at risk for vitamin D intoxication as vitamin D supplementation is routinely prescribed and usually administered as a compounded preparation that includes vitamin A, D, E, and K (ADEK) supplements. A recent report of 10-year surveillance in CF patients found a $5 \%$ prevalence rate of overdosage (6).

Here, we report the presence of a severe vitamin $\mathrm{D}$ intoxication in a young girl with $\mathrm{CF}$ due to a manufacturing error in the compounded preparation of the vitamin ADEK supplement. This case report raises awareness to urgently 
assess for vitamin D intoxication in those presenting with vague abdominal symptoms, fatigue, and polydipsia and reaffirms the international guidelines to regularly measure serum calcium and 25-hydroxyvitamin D (25(OH)D) concentrations in children with $\mathrm{CF}$ receiving ADEK supplements.

\section{Case presentation}

A 4-year-old girl with $\mathrm{CF}$ and exocrine pancreatic insufficiency, presented with suprapubic abdominal pain, decreased appetite, constipation, fatigue, weight loss, polyuria, and polydipsia for 10 days. Her current medications were colistimethate sodium and hypertonic saline aerosols, corticoid nasal spray, pancreatin capsules - a preparation of pancreatic enzymes, and a compounded vitamin ADEK preparation. Family history was unremarkable. Her fluid intake was $~ 1.5 \mathrm{~L}$ per day and she passed large volumes of urine during both daytime and nighttime. She lost $2.1 \mathrm{~kg}$ in weight over the past 2.5 months and complained of decreased appetite, constipation, and frequent nocturnal leg cramps. The parents initially linked the above symptoms with schoolrelated anxiety but finally consulted a paediatrician upon aggravation.

On examination, the mental and hydration status were normal, the patient was afebrile, the mean blood pressure was $90 \mathrm{~mm} \mathrm{Hg}$, and the pulse rate was 100 b.p.m. Body weight was $15.1 \mathrm{~kg}$ (Z score -0.39), height was $105 \mathrm{~cm}$ (Z score 0,89 ), and BMI (the weight in kilograms divided by the square of the height in meters) was $13.7 \mathrm{~kg} / \mathrm{m}^{2}$. The abdomen was soft and nontender, but a vague mass was palpated in the left iliac fossa. On electrocardiography, a sinus tachycardia of 121 b.p.m. was noted. Clinical suspicion for CF-related diabetes was raised but serum glucose was normal and no glucosuria was found. The laboratory results are shown in Table 1 . The elevated serum calcium and $25(\mathrm{OH}) \mathrm{D}$ were suggestive of a vitamin D intoxication. The last laboratory control 11 months prior to presentation showed a serum calcium level of $2.48 \mathrm{mmol} / \mathrm{L}$ and $25(\mathrm{OH}) \mathrm{D}$ of $30.1 \mu \mathrm{g} / \mathrm{L}$. No other vitamin and nutritional supplements or fish oil preparations were used, but a new batch of vitamin ADEK supplements was started 2 weeks earlier. The prescription of ADEK supplements clearly read 800 IU vitamin D per capsule, but a re-examination of the preparation schedule by the pharmacist revealed a manufactured dose of $8000000 \mathrm{IU}$ vitamin $\mathrm{D}$, that is, a 10000 -fold of the prescribed dose due to a dilution error.

Intravenous hyperhydration was started immediately and loop diuretics were administered. After $31 \mathrm{~h}$, calcium had decreased only slightly $(-0.22$ to $4.57 \mathrm{mmol} / \mathrm{L})$ and a single dose of pamidronate disodium $(1 \mathrm{mg} / \mathrm{kg})$ was infused. The symptoms resolved gradually and after 4 days serum calcium was normal (Fig. 1A). The urinary fractional calcium excretion could be increased transiently by hyperhydration and furosemide treatment (Fig. 1B). Serum 25(OH)D progressively decreased but remained elevated after 4 months (Fig. 1C). Ultrasonography of kidneys and heart showed no abnormalities at first. Ultrasound of the kidneys 6 months after the event showed bilateral nephrolithiasis.

\section{Discussion}

In this case report, we describe the clinical presentation and disease course of severe vitamin D intoxication in a young child with CF. A 10 000-fold dosage error of vitamin D contained in the compounded ADEK vitamin supplement caused symptomatic hypercalcemia. Hyperhydration, loop

Table 1 Laboratory data.

\begin{tabular}{l}
\hline Variable \\
\hline Serum \\
Ca $(\mathrm{mmol} / \mathrm{L})$ \\
Phosphate $(\mathrm{mmol} / \mathrm{L})$ \\
Creatinine $(\mathrm{mg} / \mathrm{dL})$ \\
PTH $(\mathrm{ng} / \mathrm{L})$ \\
$25(\mathrm{OH}) \mathrm{D}(\mu \mathrm{L} / \mathrm{L})$ \\
$1,25(\mathrm{OH}) 2 \mathrm{D}(\mathrm{pmol} / \mathrm{L})$ \\
Urine \\
Creatinine $(\mathrm{mg} / \mathrm{dL})$ \\
Ca $(\mathrm{mmol} / \mathrm{L})$ \\
Phosphate $(\mathrm{mmol} / \mathrm{L})$ \\
Urinary Ca excretion (mg calcium/mg creatinine) \\
Fractional Ca excretion (\%) \\
Tubular reabsorption phosphate $(\%)$ \\
\hline
\end{tabular}

https://edm.bioscientifica.com/

\begin{tabular}{ccc}
\hline Reference range & & At admission \\
$2.2-2.7$ & & 4.99 \\
$1.05-1.8$ & 1.61 \\
$0.26-0.42$ & 0.5 \\
$15-65$ & 8.8 \\
$20-50$ & 1675 \\
$36.5-216.2$ & $>360$ \\
& & 13 \\
& 20 & 3.4 \\
& 15.4 \\
& 0.2 & 1.73 \\
& 2.62 \\
& & 63.2 \\
\hline
\end{tabular}



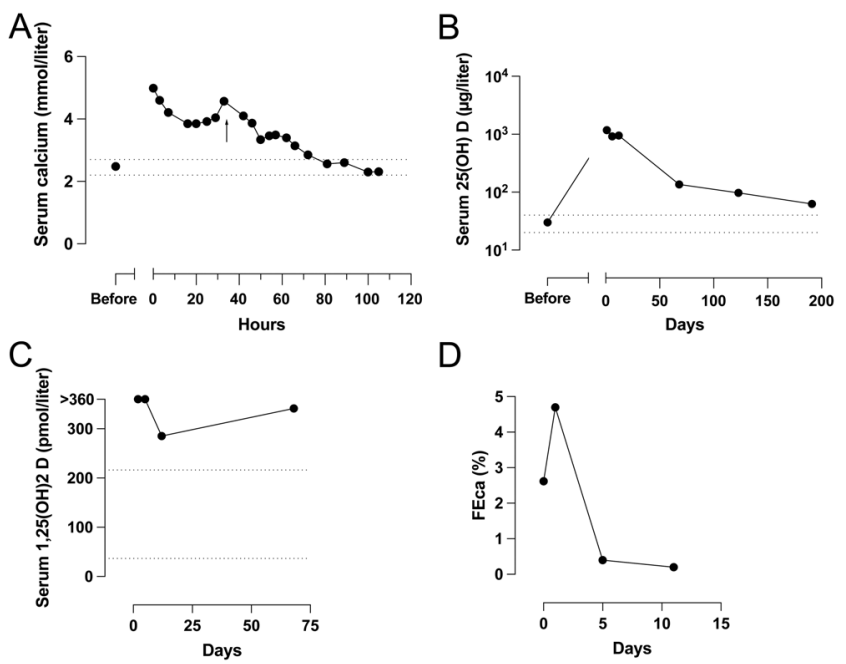

$\mathrm{D}$

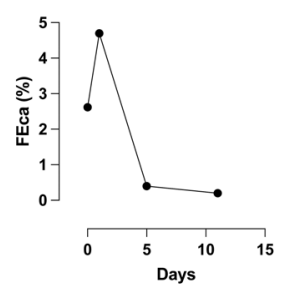

Figure 1

Kinetics of calcium and vitamin D levels. (A) Serum calcium levels, (B) serum 25(OH)D levels, (C) serum 1,25(OH)2D, and (D) fractional excretion of calcium at admission and following treatment. Arrow indicates administration of pamidronate disodium. Dotted lines indicate reference ranges.

diuretics, and bisphosphonate infusion were needed for rapid normalization of serum calcium, but 25(OH)D levels remained elevated for over 2 months.

Polyuria, polydipsia, weight loss, decreased appetite, and abdominal pain in children with $\mathrm{CF}$ is suspect for diabetes mellitus, but in general CF-related diabetes does not occur before the age of 10 years. In our case, diabetes mellitus was excluded by normal plasma and urine glucose values. The presentation of acute severe hypercalcemia or diabetes insipidus is remarkably similar and both should be included in the differential diagnosis of recent onset polydipsia and polyuria (8). Because of the absence of excessive polyuria or nocturia, no preference for cold water, no drinking at night, and normal serum sodium and serum osmolality, the diagnosis of diabetes insipidus was considered unlikely (9). Vitamin D intoxication should be the first diagnosis in hypercalcemic CF patients receiving vitamin ADEK supplements. It is unclear why most dosage errors in the ADEK preparation are made in the vitamin $\mathrm{D}$ compound. This predisposition could be related to the need for larger dilution of the stock product as compared to the other liposoluble vitamins during manufacturing. Physicians should be cautious with compounded vitamin D preparations and ensure follow-up on serum calcium and 25(OH)D levels to avoid iatrogenic toxicity. High serum calcium and 25(OH)D levels suppressed parathyroid hormone, hypercalciuria, and raised urinary calcium/ creatinine ratio should raise suspicion for vitamin D toxicity (6). Although symptomatic vitamin D intoxication with severe hypercalcemia is described on doses of $50000 \mathrm{IU} /$ day, most reports in infants and young children document extremely high doses ranging from 240000 to $4500000 \mathrm{IU}$, or approximately $40000-560000 \mathrm{IU} / \mathrm{kg}$. These excessive doses resulted in serum 25(OH)D levels between 250 and $670 \mu \mathrm{g} / \mathrm{L}$ and symptomatic hypercalcemia $(2,5,7)$. In this report, the patient received an excessive dose of 530000 $\mathrm{IU} / \mathrm{kg}$ for 2 weeks resulting in a peak serum 25(OH)D level of $1675 \mu \mathrm{g} / \mathrm{L}$. We suspect that the low BMI of our patient might result in this extremely high level since the size of the adipose mass relates to its capacity to store vitamin D (10). Hence a lower body fat size is likely to result in higher serum 25(OH)D levels upon overdose. As children with CF in general have lower body fat stores, we expect $25(\mathrm{OH}) \mathrm{D}$ levels to normalize faster as compared to healthy children, in whom normalization sometimes takes in general 3 months $(2,10,11)$. On the other hand, the variable relationship between the amount of vitamin $\mathrm{D}$ ingested and levels of circulating 25(OH)D could be explained by the length of time between the vitamin $\mathrm{D}$ ingestion and laboratory evaluation as well as in polymorphisms in genes relating to $25(\mathrm{OH}) \mathrm{D}$ metabolism (2).

High serum concentrations of $25(\mathrm{OH}) \mathrm{D}$ or free $1,25(\mathrm{OH}) 2 \mathrm{D}$ result in hypercalcemia by increased intestinal calcium absorption and bone resorption. The first step in treatment is to stop all calcium and vitamin D supplements and start a calcium and vitamin D-restricted diet. In severe hypercalcemia, i.v. hyperhydration with normal saline at 1.5-2.5 times the daily maintenance fluid requirements to increase the glomerular filtration rate and calcium excretion should be started rapidly. In addition, loop diuretics such as furosemide at $1-2 \mathrm{mg} / \mathrm{kg} /$ dose to increase renal calcium excretion can be an additional useful therapy $(2,7,12)$. Bisphosphonates, preferably Pamidronate IV $(0.5-1 \mathrm{mg} / \mathrm{kg} /$ dose with at least 48 hours between doses), are the treatment of choice in symptomatic vitamin D intoxication. They decrease calcium by inhibiting osteoclastic action and bone resorption and hereby counteract the increased bone resorption caused by vitamin D excess $(3,13)$. As seen in this case, normalization of serum calcium is seen after 3-5 days. Adverse effects of bisphosphonates include fever, bone, joint or muscle pain, hypocalcemia, hypophosphatemia, and renal toxicity $(2,7,13)$. Glucocorticoids are a treatment alternative as they decrease renal tubular reabsorption and intestinal absorption of calcium. However, the calcium-lowering effect of glucocorticoids may take up to three days and rarely normalizes calcium levels. Its low efficiency in severe hypercalcemia precludes its use in monotherapy (7). When hypercalcemia is life-threatening and refractory to 
the above treatment modalities, haemodialysis should be considered $(2,7)$.

Vitamin D intoxication should be suspected in children with CF receiving a compounding prepared vitamin D preparation when presenting with decreased appetite, constipation, fatigue, nycturia, and weight loss. In severe hypercalcemia, the first treatment step is urgent rehydration combined with bisphosphonate infusion and dietary restrictions on calcium and vitamin D intake.

\section{Declaration of interest}

The authors declare that there is no conflict of interest that could be perceived as prejudicing the impartiality of this case report.

\section{Funding}

The authors received no specific funding for this work. W S holds a senior clinical investigator grant from the Research Foundation Flanders (File number: 77833).

\section{Patient consent}

Written informed consent for publication of their clinical details was obtained from the parents of the patient.

Ethics approval and consent to participate

The study was approved by the UZ Brussel ethical committee (File 2021326, BUN 1432021000619).

\section{Author contribution statement}

E N wrote the original draft. W S supervised the writing. All other authors reviewed and revised the manuscript. $E D$ W,$B$, and $W \mathrm{~S}$ treated the patient. All authors approved the final manuscript.

\section{References}

1 Garbim BB, D’Avila L, Pinto Rigatto SZ, Rosana da Silva Quadros K, Santoro Belangero VM \& Bueno de Oliveira R. Hypercalcemia in children: three cases report with unusual clinical presentations.
Journal Brasileiro de Nefrologia 201739 213-216. (https://doi. org/10.5935/0101-2800.20170036)

2 Vogiatzi MG, Jacobson-Dickman E, DeBoer MD \& Drugs, and Therapeutics Committee of the Pediatric Endocrine Society. Vitamin D supplementation and risk of toxicity in pediatrics a review of current literature. Journal of Clinical Endocrinology and Metabolism 201499 1132-1141. (https://doi.org/10.1210/jc.2013-3655)

3 Nimesh M, Singh P, Jhamb U \& Dubey AP. An unsuspected pharmacological vitamin $\mathrm{D}$ toxicity in a child and its brief review of literature. Toxicology International 201522 167-169. (https://doi. org/10.4103/0971-6580.172284)

4 Galior K, Grebe S \& Singh R. Development of vitamin D toxicity from overcorrection of vitamin D deficiency: a review of case reports. Nutrients 201810 953. (https://doi.org/10.3390/nu10080953)

5 Conti G, Chiricco V, Lacquaniti A, Silipigni L, Fede C, Vitale A \& Fede C. Vitamin D intoxication in two brothers: be careful with dietary supplements. Journal of Pediatric Endocrinology and Metabolism 201427 763-767. (https://doi.org/10.1515/jpem-2013-0220)

6 Planté-Bordeneuve T, Berardis S, Bastin P, Gruson D, Henri L \& Gohy S. Vitamin D intoxication in patients with cystic fibrosis: report of a single center cohort. Scientific Reports 202111 7719. (https://doi. org/10.1038/s41598-021-87099-w)

7 Demir K, Döneray H, Kara C, Atay Z, Cetinkaya S, Cayir A, Anik A, Eren E, Uçaktürk A, Can Yılmaz G, et al. Comparison of treatment regimens in management of severe hypercalcemia due to vitamin D intoxication in children. Journal of Clinical Research in Pediatric Endocrinology 201911 140-148. (https://doi.org/10.4274/jcrpe. galenos.2018.2018.0131)

8 Mackie AD, Thornton SJ \& Edenborough FP. Cystic fibrosis-related diabetes. Diabetic Medicine 200320 425-436. (https://doi.org/10.1046/ j.1464-5491.2003.00924.x)

9 Christ-Crain M. EJE AWARD 2019: New diagnostic approaches for patients with polyuria polydipsia syndrome. EJE 2019. European Journal of Endocrinology 2019181 R11-R21. (https://doi.org/10.1530/EJE-19. 0163)

10 Arunabh S, Pollack S, Yeh J \& Aloia JF. Body fat content and 25-hyrdroxyvitamin D levels in healthy women. Journal of Clinical Endocrinology and Metabolism 200388 157-161. (https://doi. org/10.1210/jc.2002-020978)

11 Mailhot G. Vitamin D bioavailability in cystic fibrosis: a cause for concern? Nutrition Reviews 201270 280-293. (https://doi.org/10.1111/ j.1753-4887.2012.00471.x

12 Kara C, Gunindi F, Astyol A \& Aydin M. Vitamin D intoxication due to an erroneously manufactured dietary supplement in seven children. Pediatrics 2014133 e240-e244. (https://doi.org/10.1542/peds.20130711)

13 Simm PJ, Biggin A, Zacharin MR, Rodda CP, Tham E, Siafarikas A, Jefferies C, Hofman PL, Jensen DE, Woodhead H, et al. Consensus guidelines on the use of bisphosphonate therapy in children and adolescents. Journal of Paediatrics and Child Health 201854 223-233. (https://doi.org/10.1111/jpc.13768)

Received in final form 14 January 2022

Accepted 20 January 2022 